Quality Improvement

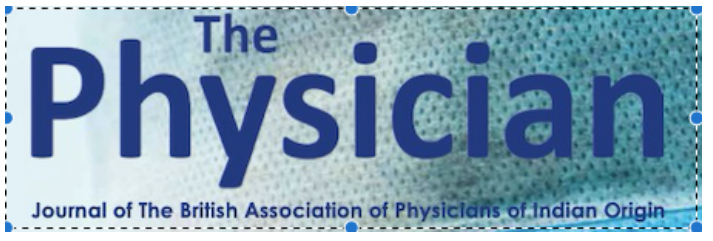

\title{
Haematuria Readmission Rates in DOAC Patients Undergoing TURP/TURBTs and Urinary Tract Biopsies - Is There any Need for a Bridging Plan?
}

Abstract

Aim - The purpose of our quality improvement project was to reduce readmission rates for haematuria in patients on direct oral anticoagulants (DOACs) who had undergone a urinary tract biopsy or resection.

Methods - For each cycle we used operating lists, pre-assessment clerking and departmental inpatient lists to identify the proportion of patients on DOACs readmitted post-operatively within 1 month from date of surgery. Cycle 1 was completed over a six-month period. We then discussed these results with a Haematologist, who advised a bridging plan with low-molecular weight heparin to mitigate the risk. Following implementation of the bridging plan, we then completed the second cycle over a three-month period.

Results - The first cycle showed that $37.5 \%(n=16)$ of all patients on DOACs who had undergone one of these procedures were readmitted with significant haematuria. After implementation of the bridging plan with Low molecular weight heparin, the second cycle showed a reduced readmission rate of $33.3 \%(n=9)$, despite a higher percentage of patients on a DOAC in this cycle.

Discussion - There was no uniform practice or protocol for restarting DOACs in our hospital. After completion of this project, a uniform protocol has been established.

Recommendations included: (1) rediscuss with a Haematologist whether further measures were needed; present our data at a regional meeting to survey protocol and practices in neighbouring hospitals. Limitations included: a small sample size; non-uniform duration of data collection per cycle; reduction of elective operative lists due to COVID-19.

Keywords: haematuria, DOACs, TURP, TURBT
Tavishi Kanwar \& Alice Li

(Joint first authors)

Kingston Hospital, London, UK

Correspondence -

tavishi.kanwar@nhs.net

Alice.li1@nhs.net

Cite as: Kanwar, T. \& Li, A. (2021)

Haematuria readmission rates in DOAC patients undergoing TURP/TURBT and urinary tract biopsies - Is there any need for a bridging plan? The Physician vol 7; Issue 2: ePub 14.12.21 DOI:

https://doi.org/10.38192/1.7.2.3

Article Information

Submitted 5.12.21

Epub 14.12.21

ISSN 


\section{Introduction}

The decision of when to offer surgical intervention and how to manage anticoagulation (AC) or antiplatelet (AP) medication in the perioperative setting is a dilemma with which surgeons are dealing with increasing frequency.[1] In an ageing population the commonest reason for long term anticoagulation includes atrial fibrillation and thromboembolic disease. Patients treated with oral anticoagulants (DOAC) had significantly greater risk of all-cause, major bleeding-related readmissions with average length of stay being significantly longer and costs significantly higher for patients on warfarin. [2] However, discontinuation of AC/AP therapy before surgery may predispose patients to thromboembolism caused by the release of tissue thromboplastins. [3]

Haematuria and urinary tract infections are common reasons for patients presenting to emergency departments or requiring readmission following urological procedures with rates around 4.4-14.4\%.[4] Hospital readmission after surgery contribute to morbidity, quality of life, healthcare costs and are a key metric for healthcare quality. Raslan et al., for instance, showed 30-day unplanned readmission rate of $4.4 \%$ over 12 months in a UK Urology department. [5] Similarly, Gore et al., reported a $31 \%$ rate of readmission within 90 days after urinary diversion [6] while Harraz et al. revealed that the development of high-grade postoperative complications was a significant predictors for readmission after surgery. [7]

The Urology department at Kingston Hospital, London noticed a number of patients on DOACs who were readmitted with haematuria following trans-urethral bladder biopsies (TURBT), transurethral resection of prostate (TURP) and urinary tract biopsies. These readmissions contributed significantly to the morbidity after the procedures. There are currently no national guidelines on bridging plans for these procedures. A systematic review of 13 trials with over 3000 patients, exploring safety of surgery in benign hyperplasia of prostate in patients on anti-platelet or anticoagulation, concluded that a bridging plan with Low molecular weight heparin (LMWH) was beneficial. [3]

\section{Aim}

The purpose of our project was to reduce readmission rates to inform whether any interventions such as a bridging plan with $\mathrm{LMWH}$ would be necessary.

\section{Methods}

Through the hospital's Information Services department, we obtained data via the procedure coding system to identify patients undergoing TURPs, TURBTs and urinary tract biopsies over a specific period of time. The timeframe for our first cycle was a six-month period between 01/07/2020 to $31 / 12 / 2020$. The timeframe for our second period was over 3 months between 01/06/2021 to 31/08/2021 (figure 1). We used pre-admission clerking documentation to establish the proportion of patients on DOACs. By reviewing inpatient departmental lists, we then identified the number of patients readmitted with haematuria from the subset of patients on DOACs within one month of the procedure.

We discussed the results from our first cycle with the local Haematologist, who advised a bridging protocol of post-operative LMWH (Fragmin $® 5000$ international units (iu) once daily for low/intermediate-risk patients and 5000 iu twice daily for high-risk patients, then to restart DOAC on day 7 post-operatively). After the first cycle, a new protocol was established where the patients on DOACs were identified by the pre-assessment team and referred to the anticoagulation department to implement the agreed bridging plan. Through review of documentation, it was confirmed that this bridging plan was successfully implemented in all subsequent patients. 
Figure 1 - Timeline of project
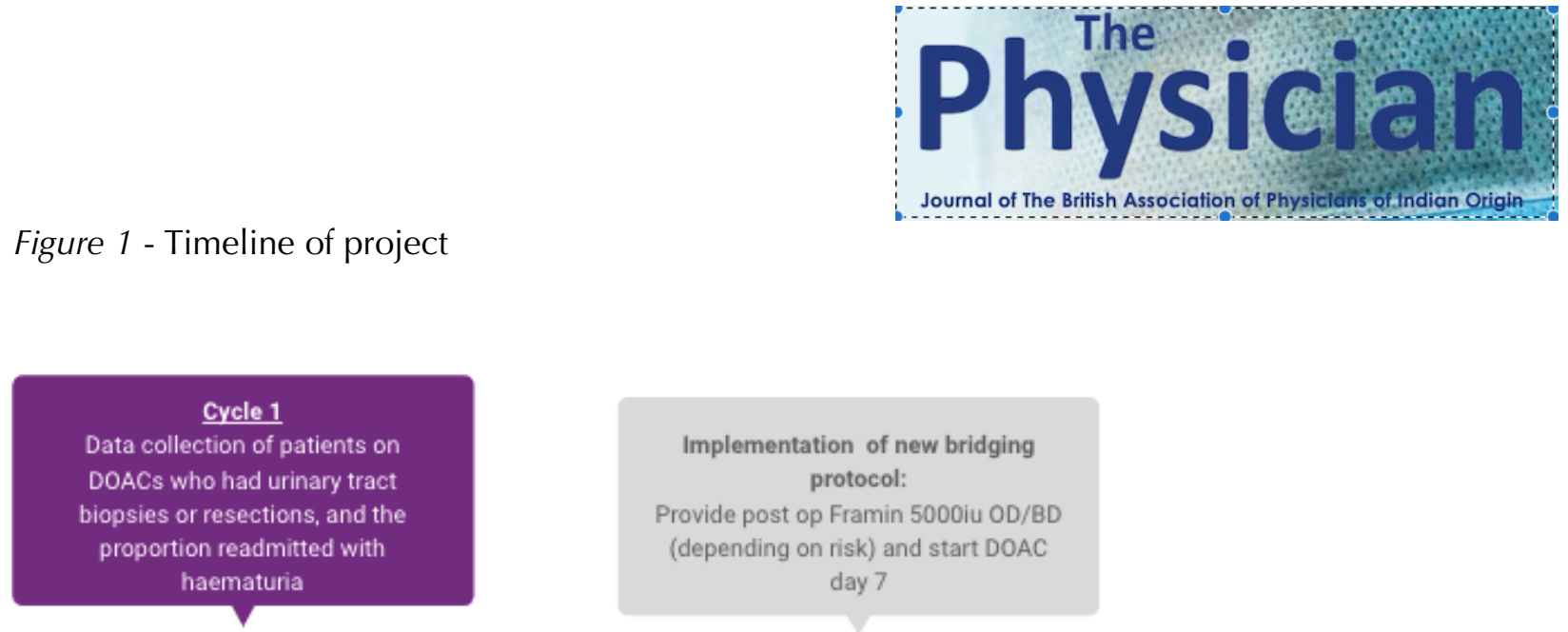

July-Dec '20

May '21
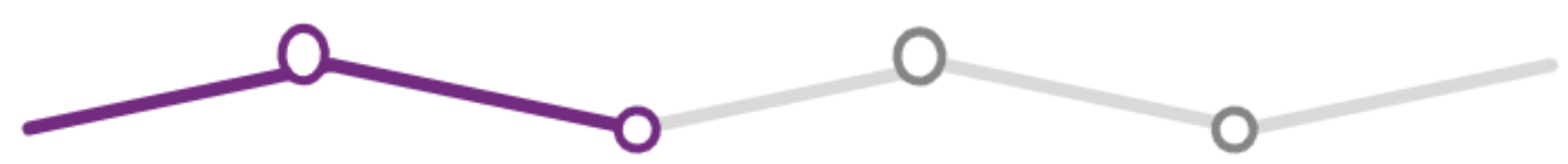

Jun-Aug'21

Initial discussions with

haematology re: possible

interventions

Cycle 2

Repeat cycle 1 using the same data collection methods

\section{Results}

From the first cycle there were a total of 146 patients who had a TURP, TURBT or urinary tract biopsy (Table 1$)$. There were 16 patients $(11.0 \%)$ on a DOAC, of whom 6 were readmitted due to haematuria following the procedure $(37.5 \%)$ (figure 2), all were male with ages between 75 and 90 years.
In the second cycle, 63 patients had one of the procedures, of whom 9 (14.3\%) were on a DOAC (table 1). Three patients were readmitted due to haematuria (33.3\%) (figure 2), 2 were male with ages between 71 and 83 years.

Interventions to manage the haematuria included 3-way catheter insertion, bladder washout and irrigation and blood transfusion. 
Table 1: Patients included in the project

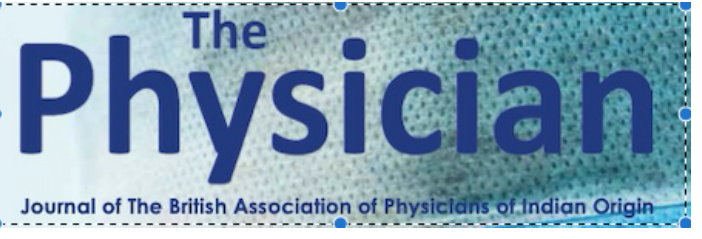

\begin{tabular}{|l|l|l|l|l|l|l|}
\hline & Total & On DOAC & $\begin{array}{l}\text { Readmission } \\
\mathbf{s}\end{array}$ & $\begin{array}{l}\text { \% patients } \\
\text { on DOAC }\end{array}$ & $\begin{array}{l}\text { \% } \\
\text { readmissions } \\
\text { on DOAC }\end{array}$ & \% total \\
\hline Cycle 1 & 146 & 16 & 6 & 10.9 & 37.5 & 4.1 \\
\hline Cycle 2 & 63 & 9 & 3 & 14.3 & 33.3 & 4.8 \\
\hline
\end{tabular}

Figure 2: Patients on DOACs and readmission rates

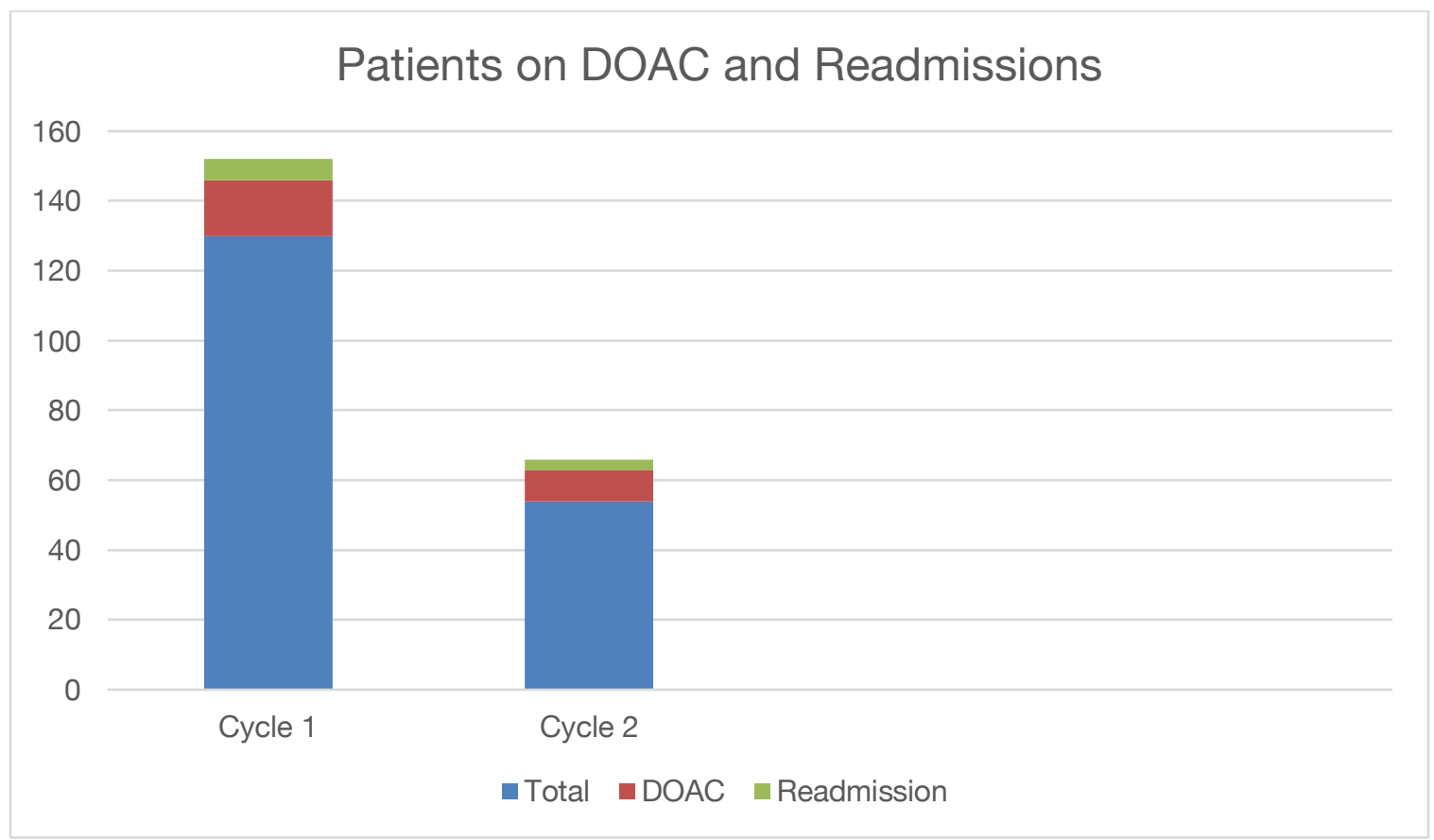

\section{Discussion}

Overall, our intervention appeared to show a small reduction in readmission rates, however there may be further room for improvement in this area, although the numbers are too small to apply statistical analysis. Prior to the intervention, there was no uniform practice in our hospital on managing patients on DOACs undergoing aforementioned procedures, with patients being restarted on the DOAC a variable number of days post-operatively. We now have uniform practice.

We recognise there is an additional cost of implementing a bridging plan with $\mathrm{LMWH}$ : patients require an additional appointment prior to procedure and require training or district nurses to administer subcutaneous injections. This requires coordination between pre-assessment, 
anticoagulation clinic, pharmacy, GP and community services. However, the predicted reduction in complications, readmission with increased length of stay is likely to mitigate this additional cost.

There is evidence that concurrent use of DOAC medication increases the morbidity of urinary tract procedures, with increased lengths of admission, larger haemoglobin drops and higher numbers of patients requiring blood transfusions. [8] [9] However, it is noted that, while there is an increased risk of complication, most are minor (Clavien-Dindo <3) and all studies felt the urinary tract procedures, including radical prostatectomies, are safe for these patients and the benefits outweigh the risks. Furthermore, one study shows no significant increase in risk of thromboembolic disease for patients on a bridging protocol. [10] Further larger trials and metaanalysis are needed in this area to inform practice.

On reflection of the results from our second cycle, we recommend rediscussing with a Haematologist and to survey other Trusts 'practice in this area, for example through presentation and discussion of this data at regional meetings.

Limitations of this project include the small sample size and lack of statistical analysis. Our data collection was of different duration for the first and second cycle; this audit could be improved by longer data collection for the second cycle. An external limiting factor was the COVID-19 pandemic, which resulted in a reduction of elective operating lists in our hospital during the period of data collection, meaning that fewer of these procedures were done compared with prepandemic. In addition, patients who had these procedures were prioritised clinically, which may have skewed the data. It would be interesting to collect data on the histology of the biopsies and tumour size to see whether there was a correlation with readmission for haematuria. This could test the hypothesis that patients with a malignancy found on biopsy, or larger tumour size are more likely to have heavier bleeding post-operatively. Previous studies have found larger tumour size to be a risk factor for post-op clot-retention ${ }^{5}$.

The next steps for this project include further cycles of data collection to increase statistical significance. We would consider collecting data in the same fashion for patients on warfarin; it has been noted in the literature that patients on warfarin are more likely to bleed post-procedure than patients on DOAC.

\section{References}

1 Heiman J, Large T, Krambeck A. Best practice in the management of benign prostatic hyperplasia in the patients requiring anticoagulation. Ther Adv Urol 2018;10:4316. doi:10.1177/1756287218807591

2 Deitelzweig S, Baker CL, Dhamane AD, et al. Comparison of readmissions among hospitalized nonvalvular atrial fibrillation patients treated with oral anticoagulants in the United States. J Drug Assess;9:87-96. doi:10.1080/21556660.2020.1750418

3 Liang X, Wu W, Huang Y, et al. Safety of Surgery in benign Prostatic Hyperplasia Patients on Antiplatelet or Anticoagulant Therapy: A Systematic Review and MetaAnalysis. Urol J 2020;18:151-9. doi:10.22037/uj.v16i7.5974

4 Palmisano F, Boeri L, Fontana M, et al. Incidence and predictors of readmission within 30 days of transurethral resection of the prostate: a single center European experience. Sci Rep 2018;8:6575. doi:10.1038/s41598-018-25069-5

5 Unplanned urology readmissions in a district general hospital: are we meeting the standard? - M Raslan, Ms Floyd, S Itam, R Mukherjee, Pp Irwin, Sb Maddineni, 2013. https://journals.sagepub.com/doi/10.1177/20 51415813487333 (accessed 14 Dec 2021).

6 Readmissions in the postoperative period following urinary diversion | SpringerLink. 
https://link.springer.com/article/10.1007\%2Fs 00345-010-0613-8 (accessed 14 Dec 2021).

7 Risk factors of hospital readmission after radical cystectomy and urinary diversion: analysis of a large contemporary series Harraz - 2015 - BJU International - Wiley Online Library. https://bjui-

journals.onlinelibrary.wiley.com/doi/10.1111 /bju.12830 (accessed 14 Dec 2021).

8 Becker B, Netsch C, Hansen J, et al. Perioperative Safety in Patient Under Oral Anticoagulation During Holmium Laser Enucleation of the Prostate. J Endourol 2019;33:219-24. doi:10.1089/end.2018.0693

9 Beckmann A, Spalteholz J, Langer F, et al. Perioperative management of direct oral anticoagulants in patients undergoing radical prostatectomy: results of a prospective assessment. World J Urol 2019;37:2657-62. doi:10.1007/s00345-019-02668-z

10 Pose R, Langer F, Tennstedt P, et al. Management of Patients Receiving Direct Oral Anticoagulants Scheduled for Radical Prostatectomy: An Update of a Prospective Assessment. Eur Urol Focus 2021;:S24054569(21)00012-2. doi:10.1016/j.euf.2021.01.011

Conflicts of interest: Nothing to declare Author contribution:

Dr Tavishi Kanwar - Foundation doctor - Project lead and first author

Dr Alice Li - Foundation doctor - Project lead and first author

Miss Rashmi Singh - Supervising Consultant 